\title{
CR-SUBMANIFOLDS OF A LOCALLY CONFORMAL KAEHLER SPACE FORM
}

\section{HASAN SHAHID}

\author{
Department of Mathematics \\ Faculty of Natural Sciences \\ Jamia Millia Islamia, New Delhi - 110025
}

(Received November 14, 1991 and in revised form May 20, 1992)

\begin{abstract}
Bejancu [1,2]) The purpose of this paper is to continue the study of $C R$ submanifolds, and in particular of those of a locally conformal Kaehler space form (Matsumoto [3]). Some results on the holomorphic sectional curvature, $D$-totally geodesic, $D^{1}$-totally geodesic and $D^{1}$-minimal $C R$-submanifolds of locally conformal Kaehler (l.c.k.)-space from $\bar{M}(c)$ are obtained. We have also discussed Ricci curvature as well as scalar curvature of $C R$-submanifolds of $\bar{M}(c)$.
\end{abstract}

KEY WORDS AND PHRASES. $C R$-submanifolds, $D$-totally goedesic, $D^{1}$-totally geodesic and minimal $C R$-submanifolds.

1991 AMS SUBJECT CLASSIFICATION CODE. 53B25.

\section{PRELIMINARIES.}

Let $\bar{M}$ be a Hermitian manifold with complex structure $J$. Let $\Omega$ denote the fundamental 2 -form of a Hermitian manifold $\bar{M}$ defined by $g(J X, Y)=\Omega(X, Y)$, where $g$ is the Hermitian metric and $X, Y$ are arbitrary vector fields on $\bar{M} . \quad \bar{M}$ is called a locally conformal Kaehler (l.c.k.) manifold [4] if there is a closed l-form called the Lee form on $\bar{M}$ such that $d \Omega=\Omega \wedge \omega$ where $d$ and $\wedge$ denoting exterior derivative operator and wedge product. In a l.c.k. manifold $\bar{M}$, we define a symmetric tensor field $P(X, Y)$ as

$$
P(Y, X)=-\left(\bar{\nabla}_{Y} \alpha\right)(X)-\alpha(X) \alpha(Y)+\frac{1}{2}\|\alpha\|^{2} g(X, Y),
$$

where $\|\alpha\|$ denotes the length of the Lee form with respect to $g$. Moreover, we assume that the tensor field $P$ is hybrid, that is,

$$
P(Y, J X)=-P(J Y, X)
$$

A l.c.k. manifold $\bar{M}$ is called a l.c.k.-space form if it has a constant holomorphic sectional curvature $c$, and will be denoted by $\bar{M}(c)$. Let $\bar{M}(c)$ be a l.c.k. - space form, and $M$ be a Riemannian manifold isometrically immersed in $\bar{M}$. We denote by $g$ the metric tensor field of $\bar{M}(c)$ as well as that induced on $M$. Let $\bar{\nabla}($ resp. $\nabla)$ be the covariant differentiation with respect to the Levi-Civita connection in $\bar{M}$ (resp. $M$ ). Then the Gauss and Weingarten formulas for $M$ are respectively given by

$$
\bar{\nabla}_{X} Y=\nabla_{X} Y+h(X, Y), \quad \bar{\nabla}_{X} N=-A_{X} N+\nabla_{X}^{\frac{1}{X}} N
$$

for any $X, Y \in T M$, where $h$ (resp. $A$ ) is the second fundamental form (resp. tensor) of $M$ and $\nabla \perp$ denotes the operator of the normal connection. Moreover

$$
g(h(X, Y), N)=g\left(A_{N} X, Y\right) .
$$


The curvature tensor $\bar{R}$ of a l.c.k. space form $\bar{M}(c)$ is given by Matsumoto [3]

$$
\begin{aligned}
\bar{R}(X, Y, Z, W) & =\frac{c}{4}[g(X, W) g(Y, Z)-g(X, Z) g(Y, W)+g(J X, W) g(J Y, Z)-g(J X, Z) g(J Y, W) \\
& -2 g(J X, Y) g(J Z, X)]+\frac{3}{4}[P(X, W) g(Y, Z)-P(X, Z) g(Y, W)+g(X, W) P(Y, Z) \\
& -g(X, Z) P(Y, W)]+\frac{1}{4}[P(X, J W) g(J Y, Z)-P(X, J Z) g(J Y, W)+g(J X, W) P(Y, J Z) \\
& -g(J X, Z) P(Y, J W)-2 P(X, J Y) g(J Z, W)-2 P(Z, J W) g(J X, Y)],
\end{aligned}
$$

where $\bar{R}(X, Y, Z, W)=g(\bar{R}(X, Y) Z, W)$ and

$$
P(X, Y)=P(Y, X), P(X, J Y)=-P(J X, Y), P(J X, J Y)=P\left(X, Y^{*}\right) .
$$

The Gauss equation is given by

$$
R(X, Y, Z, W)=\bar{R}(X, Y, Z, W)+g(h(X, W), h(Y, Z))-g(h(X, Z), h(Y, W)),
$$

where $R($ resp. $\bar{R}$ ) is the curvature of $M$ and (resp. $\bar{M}(c)$ ).

DEFINITION 1.1. A submanifold $M$ of a 1.c.k. space form $\bar{M}(c)$ is called a $C R$-submanifold if there exists a differentiable distribution $D: x \rightarrow D_{x} \subset T_{x} M$ on $M$ satisfying the following condition:

(i) $D$ is holomorphic i.e. $J D_{x}=D_{x}$ for each $x \in M$ and

(ii) the complementary orthogonal distribution $D^{\perp}: x \rightarrow D_{x}^{\perp} \subset T_{x} M$ is totally real, i.e. $J D_{x}^{\perp} \subset T_{x}^{\perp} M$ for each $x \in M$.

For any vector field $X$ tangent to $M$, we put

$$
X=T X+F X
$$

where $T X$ and $F X$ belong to the distribution $D$ and $D^{\perp}$ respectively.

2. SECTIONAL CURVATURE OF CR-SUBMANIFOLDS.

Let $M$ be a $C R$-submanifold of a 1.c.k. space form $\bar{M}(c)$. Then using Gauss equation (1.6), the curvature tensor of $M$ is given by

$$
\begin{aligned}
R(X, Y, Z, W)=\frac{c}{4}[g(X, W) g(Y, Z)-g(X, Z) g(Y, W)+g(J T X, W) g(J T Y, Z)-g(J T X, Z) g(J T Y, W) \\
-2 g(J T X, Y) g(J T Z, W)]+\frac{3}{4}[P(X, W) g(Y, Z)-P(X, Z) g(Y, W)+g(X, W) P(Y, Z)-g(X, Z) P(Y, W)] \\
+\frac{1}{4}[P(X, J T W) g(J T Y, Z)-P(X, J T Z) g(J T Y, W)+g(J T X, W) P(Y, J T Z)-g(J T X, Z) P(Y, J T W) \\
-2 g(J T Z, W) P(X, J T Y)-2 P(Z, J T W) g(J T X, Y)]+g(h(X, W), h(Y, Z))-g(h(X, Z), h(Y, W))
\end{aligned}
$$

for $X, Y, Z, W \in T M$.

Let $\bar{H}(X)$ be the holomorphic sectional curvature of $M$ determined by a unit vector $X$ and $J X$. Then from (1.5) we have

$$
\bar{H}(X)=\bar{R}(X, J X, J X, X)=-\frac{c}{2}+\frac{7}{4} P(X, X) .
$$

Now suppose that $\bar{K}(X \wedge Y)$ be the sectional curvature of $\bar{M}$ determined by a unit vector $X$ and $J X$. Then from (1.5) we have

$\bar{K}(X \wedge Y)=\bar{R}(X, Y, Y, X)=\frac{c}{4}\left[1+g(J X, Y)^{2}+2 g(J X, Y)\right]+\frac{3}{4}[P(X, X)+P(Y, Y)]+P(X, J Y) g(J X, Y)$.

Next, suppose that $K(X \wedge Y)$ be the sectional curvature of $M$ determined by orthonormal tangent vectors $\{X, Y\}$ of $M$. Then using (1.6) and (2.3), we have

$$
\begin{gathered}
K(X \wedge Y)=\frac{c}{4}\left[1+g(J T X, Y)^{2}+2 g(J T X, Y)\right]+\frac{3}{4}[P(X, X)+P(Y, Y)]+P(X, J T Y) g(J T X, Y) \\
+g(h(X, X), h(Y, Y))-\|h(X, Y)\|^{2},
\end{gathered}
$$

for all $X, Y$ tangent to $M$. From this, we have

PROPOSITION 2.1. Let $M$ be a $C R$-submanifold of a l.c.k. space form $\bar{M}(c)$. If $M$ is totally geodesic in $\bar{M}(c)$, then the sectional curvature of $M$ is given by

$$
K(X \wedge Y)=\frac{c}{4}\left[1+g(J T X, Y)^{2}+2 g(J T X, Y)\right]+\frac{3}{4}[P(X, X)+P(Y, Y)]+P(X, J T Y) g(J T X, Y)
$$

for all $X, Y$ tangent to $M$. 
DEFINITION 2.1. A $C R$-submanifold $M$ of a l.c.k. space form $\bar{M}(c)$ is said to be $D$-totally (resp. $D^{\perp}$-totally geodesic) if $h(X, Y)=0($ resp. $h(Z, W)=0)$ for all $X, Y \in D,\left(Z, W \in D^{\perp}\right.$ ).

Thus as an immediate consequence of $(2.5)$ we have

COROLLARY 2.2. Let $M$ be a $C R$-submanifold of a l.c.k. space form $\bar{M}(c)$. If $M$ is $D^{\perp}$. totally geodesic in $\bar{M}(c)$, then the sectional curvature of $M$ is given by

$$
K(X \wedge Y)=\frac{c}{4}+\frac{3}{4}[P(X, X)+P(Y, Y)] \quad \text { for all } X, Y \in D .
$$

The holomorphic sectional curvature $H$ of $M$ determined by a unit vector $X \in D$ is the sectional curvature determined by $\{X, J X\}$. Hence from (2.2), we have

$$
H(X)=-\frac{c}{2}+\frac{7}{4} P(X, X)+g(h(X, X), h(J X, J X))-\|h(X, J X)\|^{2} .
$$

LEMMA [1]. Let $M$ be a $C R$-submanifold of a Kaehler manifold $\bar{M}$. Then the holomorphic distribution $D$ is involutive if and only if

$$
h(J X, Y)=h(X, J Y), \quad \forall X, Y \in D .
$$

Making use of (2.8) in (2.7), we have

PROPOSITION 2.3. Let $M$ be a $C R$-submanifold of a l.c.k.-space form $\bar{M}(c)$ with involutive distribution $D$, then

$$
H(X) \leq \frac{7}{4} P(X, X), \quad \forall X \in D .
$$

Moreover from (2.7), we have

PROPOSITION 2.4. A $C R$-submanifold $M$ of a l.c.k. space form $\bar{M}(c)$ is $D$-totally geodesic if and only if the following conditions are satisfied:

(a) the holomorphic distribution $D$ is involutive, and (b) $H(X)=\frac{7}{4} P(X, X)-\frac{c}{2}, \quad \forall X \in D$.

Let $\left\{E_{1}, E_{2}, \cdots, E_{m}\right\}$ be a local field of orthogonal frames of $M$ such that $\left\{E_{1}, E_{2}, \cdots, E_{P}, E_{P+1}=J E_{1}, \cdots, E_{2 P}=J E_{P}\right\}$ (resp. $\left\{E_{2 P+1} \cdots E_{2 P+q}\right\}$ ) is a local field of frames in $D$ (resp. $D^{\perp}$ ).

DEFINITION 2.2. A $C R$-submanifold $M$ is called $D$-minimal (resp. $D^{\perp}$-minimal) if

Thus we have,

$$
\sum_{i=1}^{2 P} h\left(E_{i}, E_{i}\right)=0,\left(\text { resp. } \sum_{i=1}^{q} h\left(E_{2 P+i}, E_{2 P+i}\right)=0\right) \text {. }
$$

PROPOSITION 2.5. Let $M$ be a $D^{\perp}$-minimal $C R$-submanifold of a l.c.k. space form $\bar{M}(c)$. Then $M$ is $D$-totally geodesic if and only if

$$
K(X \wedge Y)=\frac{1}{4}[c+3(P(X, X)+P(Y, Y))], \quad \forall, X, Y \in D .
$$

\section{RICCI TENSOR AND SCALAR CURVATURE OF CR-SUBMANIFOLDS.}

Let $S$ be the Ricci tensor and $\rho$ the scalar curvature of $M$. Then

$$
S(X, Y)=\sum_{i} R\left(E_{i}, X ; Y, E_{i}\right), \quad \rho=\sum_{j} S\left(E_{j}, E_{j}\right),
$$

for any vector fields $X, Y$ tangent to $M$. By the straight forward calculation from (2.1), we get

$$
\begin{gathered}
S(X, Y)=\frac{c}{4}(m+2) g(X, Y)+\frac{3}{4} \sum_{i=1}^{m}\left\{P\left(E_{i}, E_{i}\right) g(X, Y)-P\left(E_{i}, Y\right) g\left(X, E_{i}\right)+m P(X, Y)-P\left(X, E_{i}\right) g\left(Y, E_{i}\right)\right\} \\
-\frac{5}{4} \sum_{i=1}^{m}\left\{P\left(J Y, E_{i}\right) g\left(J X, E_{i}\right)+P\left(J X, E_{i}\right) g\left(J Y, E_{i}\right)\right\}+\sum_{i=1}^{m}\left\{g\left(h(X, Y), h\left(E_{i}, E_{i}\right)\right)-g\left(h\left(E_{i}, X\right), h\left(E_{i}, Y\right)\right)\right\},
\end{gathered}
$$

since $g\left(J T E_{i}, E_{i}\right)=0$.

The scalar curvature is given by

$$
\rho=\frac{c}{4} m(m+2)+\sum_{i, j=1}^{m}\left\{g\left(h\left(E_{j}, E_{j}\right), h\left(E_{i}, E_{i}\right)\right)-g\left(h\left(E_{i}, E_{j}\right), h\left(E_{i}, E_{j}\right)\right)\right\} .
$$

Thus we have 
PROPOSITION 3.1. Let $M$ be a minimal $C R$-submanifold of a l.c.k. space form, then we have

(a) $S(X, Y)-\frac{c}{4}(m+2) g(X, Y)-\frac{3}{4} \sum_{i=1}^{m}\left\{P\left(E_{i}, E_{i}\right) g(X, Y)-P\left(E_{i}, Y\right) g\left(X, E_{i}\right)+m P(X, Y)-P\left(X, E_{i}\right) g\left(Y, E_{i}\right)\right\}$

$$
+\frac{5}{4} \sum_{i=1}^{m}\left\{P\left(J Y, E_{i}\right) g\left(J X, E_{i}\right)+P\left(J X, E_{i}\right) g\left(J Y, E_{i}\right)\right\}
$$

is semi-definite for all $X, Y \in D$.

(b) $\rho \leq \frac{c}{4} m(m+2)$.

Similarly we have:

PROPOSITION 3.2. Let $M$ be a minimal $C R$-submanifold of a l.c.k.-space form. Then $M$ is totally geodesic if and only if

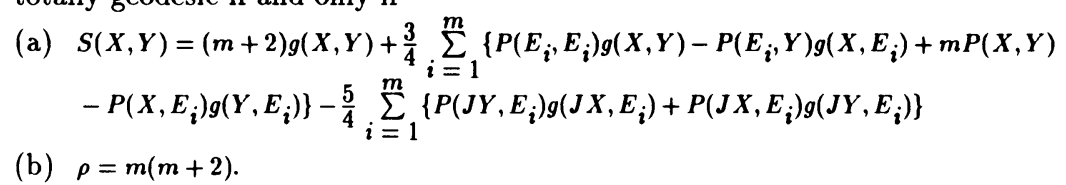

ACKNOWLEDGEMENT. The author expresses his sincere thanks to the referee for his suggestions.

\section{REFERENCES}

1. BEJANCU, A., CR-submanifolds of a Kaehler manifold I, Amer. Math. Soc. 69 (1978), 135-142.

2. BEJANCU, A., CR-submanifolds of a Kaehler manifold II, Trans. Amer. Soc. 250 (1979), 333-345.

3. MATSUMOTO, K., On CR-submanifolds of locally conformal Kaehler manifold, J. Korean Math. Soc. 21 (1984).

4. VAISMAN, I., On locally and globally conformal Kaehler manifolds, Trans. Amer. Math. Soc. $\underline{262}(1980), 533-542$.

5. CHEN, B.Y., CR-submanifolds of a Kaehlerian manifold I, J. Diff. Geom. 16 (1981), 305-322.

6. YANO, K. \& KON, M., Differential geometry of CR-submanifolds, Geometrical Dedicata 10 (1981), 369-391. 


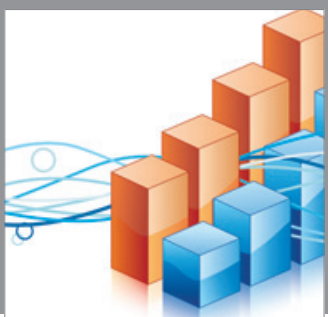

Advances in

Operations Research

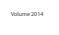

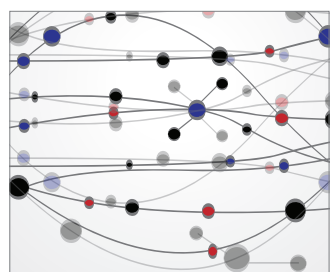

\section{The Scientific} World Journal
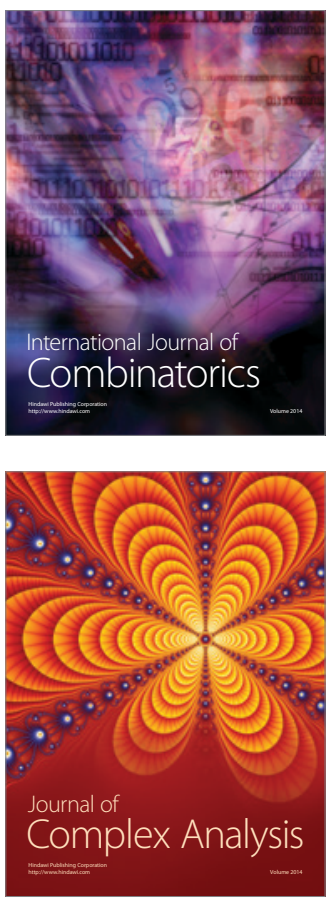

International Journal of

Mathematics and

Mathematical

Sciences
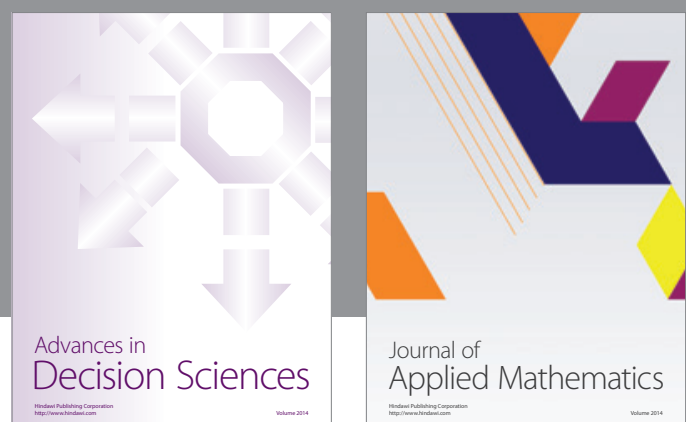

Journal of

Applied Mathematics
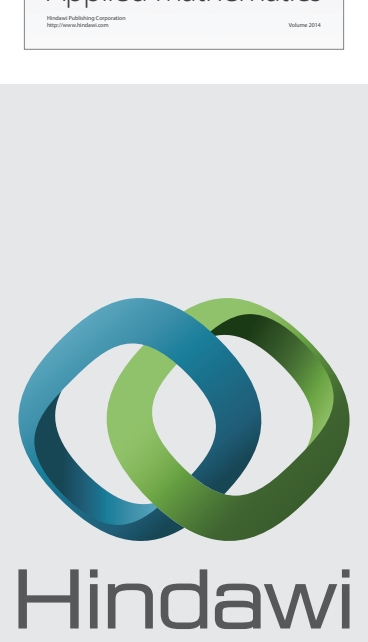

Submit your manuscripts at http://www.hindawi.com
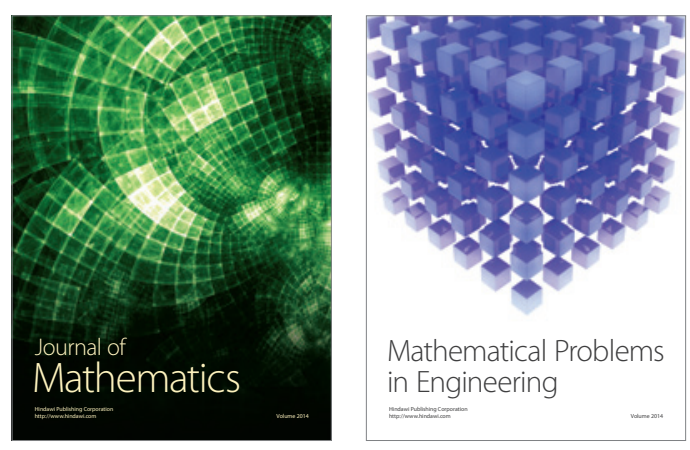

Mathematical Problems in Engineering
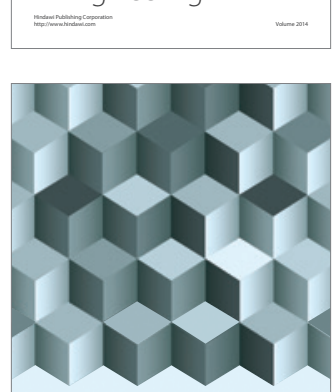

Journal of

Function Spaces
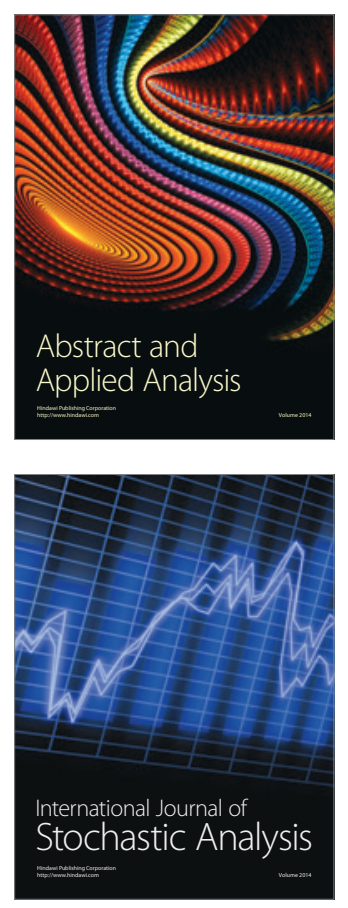

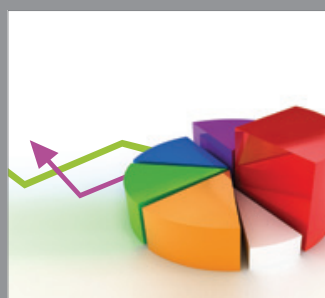

ournal of

Probability and Statistics

Promensencen
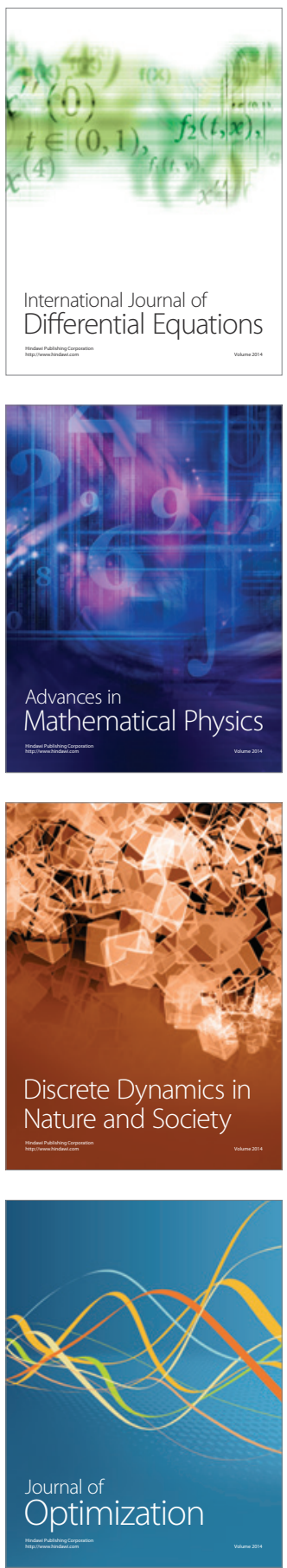